\title{
OPEN A two-way 224-Gbit/s PAM4-based fibre-FSO converged system
}

\author{
Hai-Han Lu ${ }^{1 凶}$, Chung-Yi Li ${ }^{2}$, Wen-Shing Tsai ${ }^{3}$, Poh-Suan Chang ${ }^{1}$, Yan-Yu Lin ${ }^{1}$, Yu-Ting Chen ${ }^{1}$, \\ Chen-Xuan Liu ${ }^{1}$ \& Ting Ko ${ }^{1}$
}

A two-way 224-Gbit/s four-level pulse amplitude modulation (PAM4)-based fibre-free-space optical (FSO) converged system through a 25-km single-mode fibre (SMF) transport with 500-m free-space transmission is successfully constructed, which adopts injection-locked vertical-cavity surface-emitting lasers with polarisation-multiplexing mechanism for a demonstration. Compared with one-way transmission, two-way transmission is an attractive architecture for fibre-FSO converged system. Two-way transmission over SMF transport with free-space transmission not only reduces the required number of fibres and the setups of free-space transmission, but also provides the advantage of capacity doubling. Incorporating dual-wavelength four-level pulse amplitude modulation (PAM4) modulation with polarisation-multiplexing mechanism, the transmission capacity of fibre-FSO converged system is significantly enhanced to $224 \mathrm{Gbit} / \mathrm{s}$ ( $56 \mathrm{Gbit} / \mathrm{s} \mathrm{PAM} 4$ / wavelength $\times 2$-wavelength $\times 2$-polarisation) for downlink/uplink transmission. Bit error rate and PAM4 eye diagrams (downstream/upstream) perform well over $25-\mathrm{km}$ SMF transport with $500-\mathrm{m}$ freespace transmission. This proposed two-way fibre-FSO converged system is a prominent one not only because of its development in the integration of fibre backbone with optical wireless extension, but also because of its advantage in two-way transmission for affording high downlink/uplink data rate with good transmission performance.

It is vitally important to provide broadband integrated services with long-haul transmission and high transmission capacity. Single-mode fibre (SMF) affords good transmission performance with low dispersion and low attenuation. Noticeable advantages are afforded by implementing the SMF to enhance the coverage area at relatively high-speed using the characteristics of optical fibre. Free-space optical (FSO) communications, which transport optical signals by laser light propagation in free-space, have obtained extensive attention for practically solving the problem of short-reach RF wireless communications ${ }^{1-4}$. Given the developments in SMF and FSO technologies, fibre-FSO converged system is expected to provide hundreds of gigabit services through the long-distance fibre transmission with long-reach FSO communication. To meet the targets of long-haul transmission and high transmission capacity, a two-way 224-Gbit/s four-level pulse amplitude modulation (PAM4) fibre-FSO converged system through $25-\mathrm{km}$ SMF transport with 500 - $\mathrm{m}$ free-space transmission is thereby established, adopting injection-locked vertical-cavity surface-emitting lasers (VCSELs) with polarisation-multiplexing mechanism. Two injection-locked VCSELs are orthogonally polarized and multiplexed with a wavelength spacing of $8 \mathrm{~nm}$. The transmission rate of two-way fibre-FSO converged system is significantly enhanced by integrating PAM4 modulation and injection-locked VCSELs with polarisation-multiplexing mechanism. Due to the enhancement ability of VCSEL's resonance frequency, injection locking technique effectively improves the modulation response of a PAM4-based fibre-FSO converged system ${ }^{5,6}$. PAM4 modulation is an effective optical mechanism for increasing the transmission rate of fibre-FSO converged system. Polarisation-multiplexing is another effective optical mechanism for enhancing the transmission rate of fibre-FSO converged system. As each wavelength is transported with orthogonal polarisation, the utilization of a polarisation-multiplexing mechanism doubles the transmission rate $^{7-10}$. Combining PAM4 modulation with polarisation-multiplexing mechanism guides a way to considerably increase the transmission rate of the fibre-FSO converged system. Compared with non-return-tozero modulation with single-wavelength and single-polarisation state, dual-wavelength PAM4 modulation with polarisation-multiplexing mechanism yields up to eight times [2 (dual-wavelength) $\times 2$ (PAM4 modulation) $\times 2$ (polarisation-multiplexing)] total transmission rate.

\footnotetext{
${ }^{1}$ Institute of Electro-Optical Engineering, National Taipei University of Technology, Taipei 10608, Taiwan. ${ }^{2}$ Department of Communication Engineering, National Taipei University, New Taipei City 23741, Taiwan. ${ }^{3}$ Department of Electrical Engineering, Ming Chi University of Technology, New Taipei City 243303, Taiwan. ${ }^{\circledR}$ email: hhlu@ntut.edu.tw
} 
Creating a simple polarisation demultiplexing mechanism is critically important for a polarisation-multiplexing fibre-FSO converged system. Uniting a tunable optical band-pass filter (TOBPF) with a polarisation beam splitter (PBS) is an easy and effective polarisation demultiplexing mechanism. It is attractive because it prevents the requirement for a complicated polarisation demultiplexing mechanism ${ }^{11,12}$. Employing a TOBPF with a PBS, the orthogonally polarized wavelengths are dramatically filtered and demultiplexed because they are located at different wavelengths and polarised with orthogonal polarisations.

Yao et al. achieved an orthogonal frequency-division multiplexing (OFDM)-based fibre-FSO converged system with heterodyne detection to increase the receiving sensitivity of FSO communication ${ }^{13}$. However, the high peak-to-average power ratio is a critical concern for an OFDM-based fibre-FSO converged system. PAM4 modulation is better than OFDM modulation because of its low power feature ${ }^{14}$. Moreover, the $1 \mathrm{~m}$ free-space transmission distance is much smaller than the corresponding value of $500 \mathrm{~m}$ adopted in this demonstrated fibre-FSO converged system. Additionally, costly external modulators and sophisticated heterodyne detection technique are required to build such expensive and complex OFDM-based fibre-FSO converged system. Transmitters with direct modulation are attractive for fibre-FSO converged system because they cost less and design easier than transmitters with external modulation. Our former study demonstrated an 800-Gbit/s wavelengthdivision-multiplexing PAM4 FSO communication with spatial light modulator-based beam tracking technology over $200 \mathrm{~m}$ free-space link ${ }^{15}$. The $200 \mathrm{~m}$ free-space transmission distance is significantly less than the associated value of $25.5 \mathrm{~km}$ (25-km SMF + 500-m free-space) developed in our proposed converged system. M. Singh et al. presented a $40-\mathrm{Gbit} / \mathrm{s}$ FSO transmission based on orbital angular momentum multiplexed beams ${ }^{16}$. Moreover, a radio over FSO link under harsh weather conditions employing mode-division multiplexing was presented ${ }^{17}$. Furthermore, a high-speed radio over FSO transmission link under dust environment conditions employing hybrid wavelength- and mode- division multiplexing was demonstrated ${ }^{18}$. However, the aggregate transmission rates of $40 \mathrm{Gbit} / \mathrm{s}, 40 \mathrm{Gbit} / \mathrm{s}$, and $160 \mathrm{Gbit} / \mathrm{s}$ and are less than the corresponding value of $224 \mathrm{Gbit} / \mathrm{s}$ operated in this demonstration. Besides, for a real implementation, building a two-way transmission rather than a one-way transmission is important. A one-way lightwave transmission system has a lot of room for improvement when considering a two-way transmission. Therefore, a two-way fibre-FSO converged system for delivering symmetric downlink/uplink data rate is constructed to meet the target. In this study, a two-way 224-Gbit/s PAM4-based fibre-FSO converged system over 25-km SMF transport with 500-m free-space transmission is constructed, which adopts directly modulated VCSELs with polarisation-multiplexing mechanism and injection-locked technique as a presentation. To the best of our knowledge, this system is the first to practically construct a two-way PAM4based fibre-FSO converged system with a high downlink/uplink transmission rate of $224 \mathrm{Gbit} / \mathrm{s}$. Since that both downlink and uplink PAM4 data signals are transmitted by the same SMF, the Rayleigh backscattering (RB) noise is considered in this two-way PAM4-based fibre-FSO converged system ${ }^{19-21}$. Additionally, the link availability of FSO communications greatly relies on weather conditions which influence the signal intensity ${ }^{22,23}$. Thus, atmospheric turbulence because of heavy rain is concerned in a 500-m free-space transmission. With a thorough examination, impressively low bit error rate (BER) of $10^{-9}$ and qualified PAM4 eye diagrams are acquired in this constructed two-way PAM4-based fibre-FSO converged system. Results show that such proposed two-way 224-Gbit/s PAM4-based fibre-FSO converged system is advantageous for developing a two-way long-haul fibre communication with free-space transmission. Moreover, it can be used in actual commercial systems to provide communication links in specific areas where RF communications are prohibited, such as in hospitals, in cabins or in oil refineries. It affords a secure link, rather than a fibre-wireless converged system, because the laser beam does not interfere with the RF signals. For future extension, a two-way fibre-FSO converged system with ultra-longhaul transmission and ultra-high transmission capacity is expected to be built by adopting PAM4 modulation and multi-stage injection-locked VCSELs ${ }^{24}$ with polarisation-multiplexing mechanism.

\section{Results}

The optical spectra of VCSEL1 and the modulation responses of VCSELs in free-running and injection-locked states with 56 Gbit/s PAM4 data signal. Figure la shows the optical spectra of VCSEL1 in free-running and injection-locked states with $56 \mathrm{Gbit} / \mathrm{s}$ PAM4 data signal. It is to be observed that as the VCSEL1 $(1533.44 \mathrm{~nm})$ is injection-locked, its optical spectrum shifts to a somewhat longer wavelength $(1533.46 \mathrm{~nm})$. With injection locking, the optical spectrum of the injection-locked laser moves to a slightly longer wavelength, instead of its original free-running wavelength. Injection locking behavior happens as a slave laser (VCSEL1) with a wavelength that is a slightly shorter than that of the master laser [distributed feedback (DFB) laser diode1 (LD1)]. In addition, it is to be found that the injection-locked VCSEL1's peak power is enhanced and the injection-locked VCSEL1's linewidth is suppressed. An injection-locked technique enhances the intensity and suppresses the linewidth of the injection-locked laser ${ }^{25,26}$. Four directly modulated DFB LDs could be used to replace four VCSELs with injection locking. Directly modulated DFB LD, however, has a large frequency chirp which degrades the transmission performance. An effective approach to reduce the frequency chirp is to operate laser with suppressed linewidth. The linewidth will be suppressed as the modulated VCSEL is injection-locked and leads to better transmission performance. Besides, the modulation responses of VCSELs in free-running and injection-locked scenarios with $56 \mathrm{Gbit} / \mathrm{s}$ PAM4 data signal are shown in Fig. 1b. With injection locking, the 3-dB modulation response is considerably enhanced from 8.4 (VCSEL1)/8.2 (VCSEL2)/8.3 (VCSEL3)/8.4 (VCSEL4) GHz to 20.6 (VCSEL1)/20.2 (VCSEL2)/20.4 (VCSEL3)/20.6 (VCSEL4) GHz. With injection locking, the difference between the injection-locked laser mode and the cavity mode is obtained, and thus produces a considerable resonance frequency improvement and a significant 3 - $\mathrm{dB}$ modulation response enhancement ${ }^{24}$. Provided that the transmission rate is $\sqrt{2}$ times the bandwidth, a PAM4-based fibre-FSO converged system with a data rate of $224 \mathrm{Gbit} / \mathrm{s}[20.2 \times \sqrt{2} \times 2($ PAM 4 modulation $) \times 2$ (dual-wavelength) $\times 2$ (polarisation-multiplexing mechanism) $>224$ ] in total can be practically constructed. 

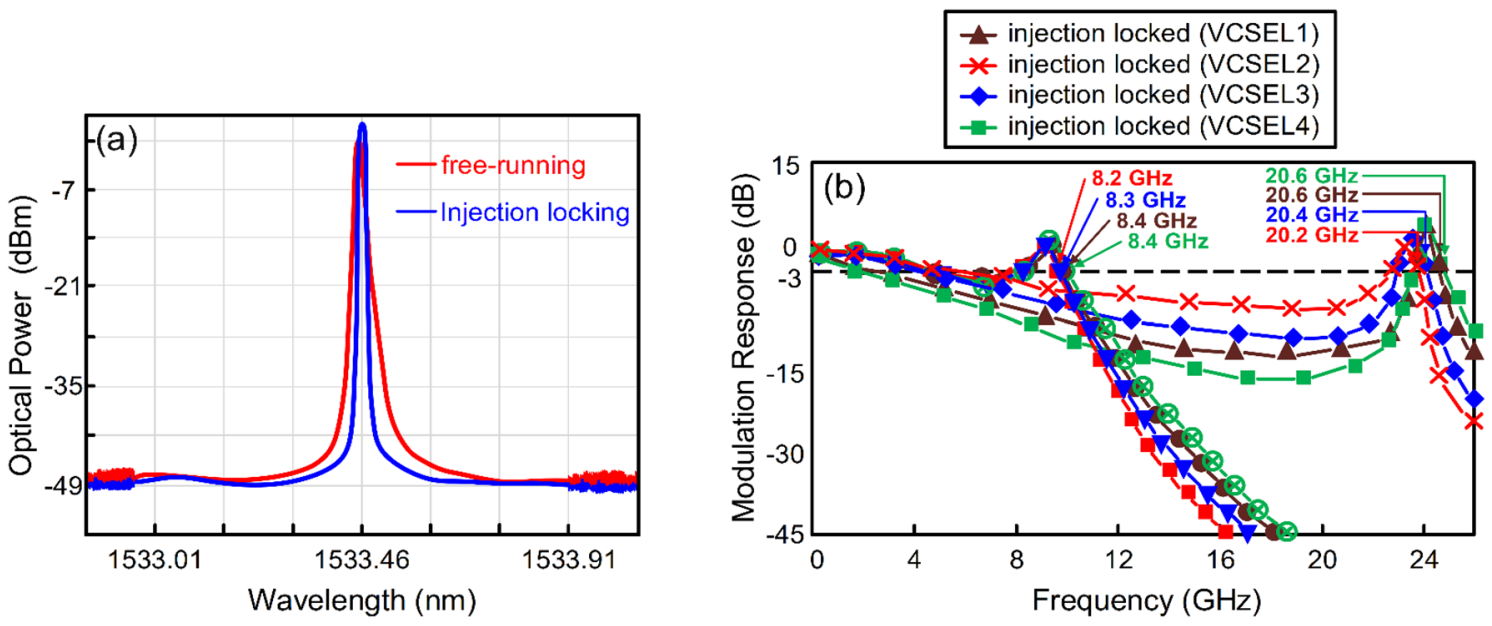

Figure 1. (a) The optical spectra of VCSEL1 in free-running and injection-locked states with 56 Gbit/s PAM4 data signal. (b) The modulation responses of VCSELs in free-running and injection-locked scenarios with $56 \mathrm{Gbit} / \mathrm{s}$ PAM4 data signal.

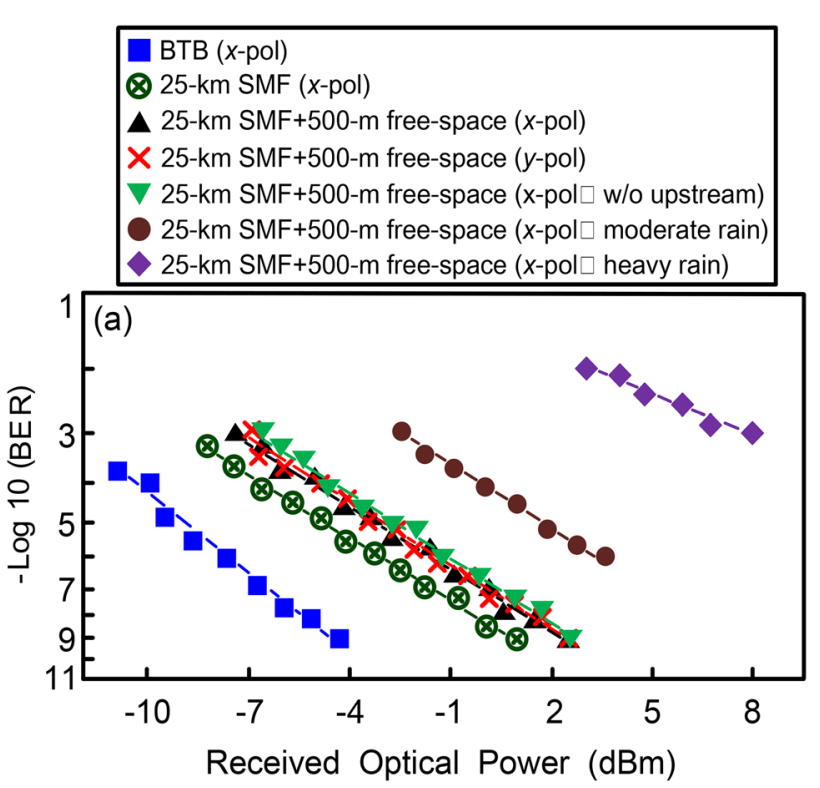

(b)

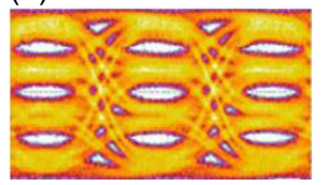

(d)

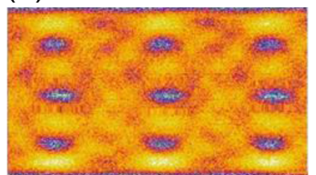

(c)

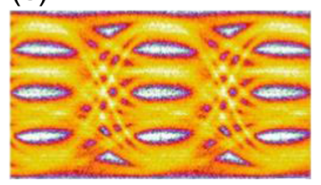

(e)

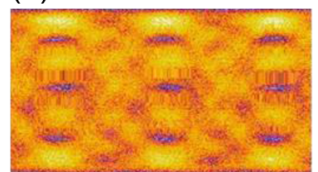

Figure 2. (a) The BER performances of 224 Gbit/s PAM4-based fibre-FSO converged system (downlink transmission) under different scenarios of back-to-back (BTB, $x$-polarisation), over 25-km SMF transport ( $x$-polarisation; sunny weather), over 25-km SMF transport with 500-m free-space transmission ( $x$ - and $y$-polarisations; sunny weather), and over 25-km SMF transport with 500-m free-space transmission ( $x$-polarisation; moderate/heavy rain). The eye diagrams of $56 \mathrm{Gbit} / \mathrm{s}$ PAM4 signal in (b) BTB state $(x$-polarisation) and that over $25-\mathrm{km}$ SMF transport with $500-\mathrm{m}$ free-space transmission ( $x$-polarisation) under (c) sunny weather, (d) moderate rain, and (e) heavy rain conditions.

BER performances of $224 \mathrm{Gbit} / \mathrm{s}$ PAM4-based fibre-FSO converged system (downlink transmission) under different scenarios and eye diagrams of $56 \mathrm{Gbit} / \mathrm{s}$ PAM4 signal in different states. Implementing a 224-Gbit/s/25.5-km (25-km SMF + 500-m free-space) PAM4-based fibre-FSO converged system is quite challenging, especially for acquiring good BER performance and competent PAM4 eye diagrams. Figure 2a presents the BER performances of $224 \mathrm{Gbit} / \mathrm{s}$ PAM4-based fibre-FSO converged system (downlink transmission) under different scenarios of back-to-back (BTB, $x$-polarisation), over 25 -km SMF transport ( $x$-polarisation; sunny weather), over $25-\mathrm{km} \mathrm{SMF}$ transport with $500-\mathrm{m}$ free-space transmission ( $x$ - and $y$-polarisations; sunny weather), and over 25-km SMF transport with 500-m free-space transmission ( $x$-polarisation; moderate/heavy rain). Under a $10^{-9} \mathrm{BER}$ operation, a 5.6 -dB power penalty appears between the BTB scenario and that over $25-\mathrm{km} \mathrm{SMF}$ transport ( $x$-polarisation; sunny weather). The $5.6 \mathrm{~dB}$ power penalty primarily arises from the fibre dispersion-induced penalty due to $25-\mathrm{km}$ SMF transport. Moreover, when the BER is $10^{-9}$, a 1.1-dB power penalty emerges between the $25-\mathrm{km}$ SMF scenario ( $x$-polarisation; sunny weather) and that 


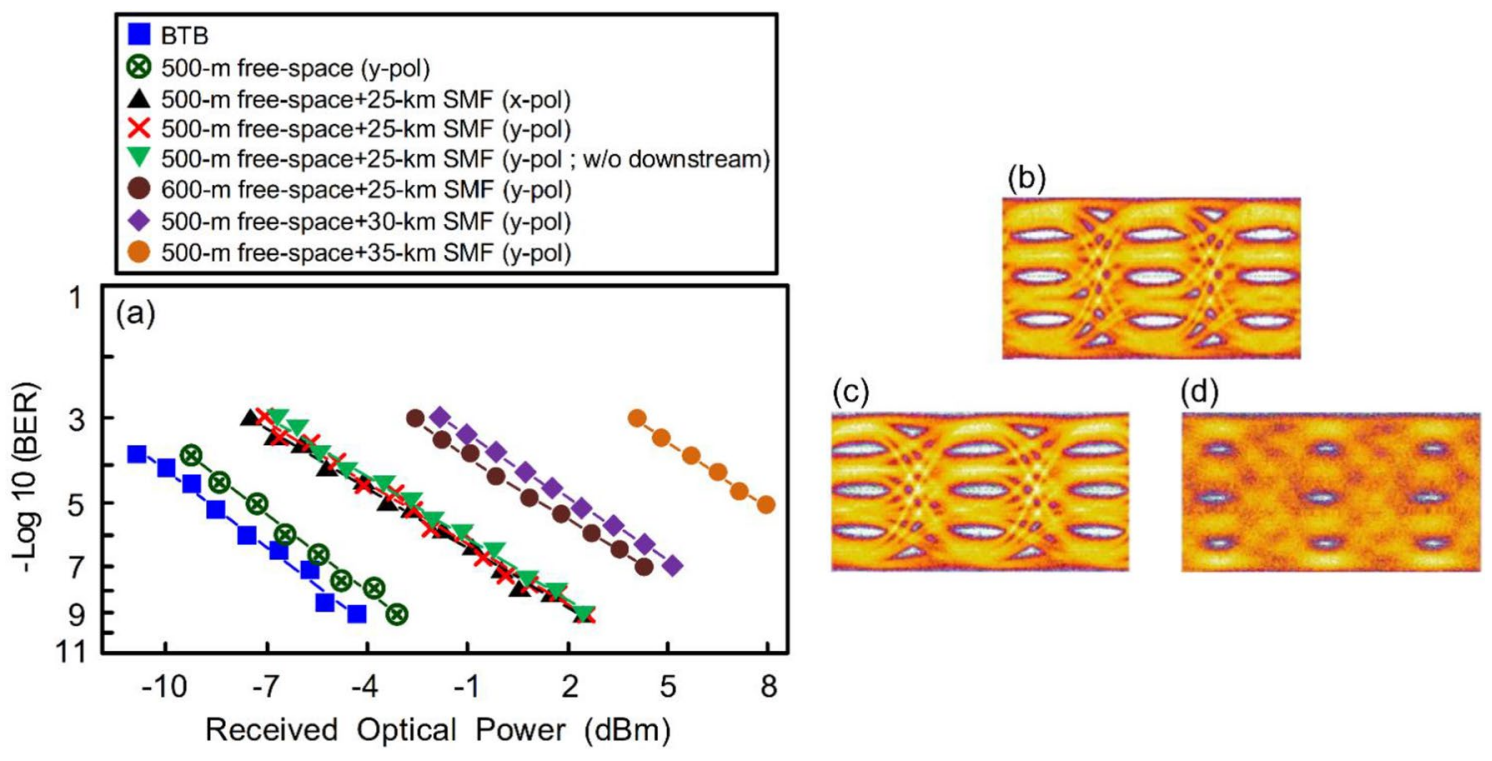

Figure 3. (a) The BER performances of $256 \mathrm{Gbit} / \mathrm{s}$ PAM4-based fibre-FSO converged system (uplink transmission) in sunny weather under the scenarios of BTB ( $y$-polarisation), over 500-m free-space transmission ( $y$-polarisation), over 500-m free-space and 25-km SMF transmissions ( $x$ - and $y$-polarisations), over 600-m free-space and 25-km SMF transmissions ( $y$-polarisation), over 500-m free-space and 30-km SMF transmissions ( $y$-polarisation), as well as over 500-m free-space and 35-km SMF transmissions ( $y$-polarisation). The eye diagrams of $56 \mathrm{Gbit} / \mathrm{s}$ PAM4 signal under the conditions (b) over 500-m free-space transmission ( $y$-polarisation), (c) over 500-m free-space and 25-km SMF transmissions ( $y$-polarisation), and (d) over 600-m free-space and 25-km SMF transmissions ( $y$-polarisation).

over 25-km SMF transport with 500-m free-space transmission ( $x$ - or $y$-polarisation; sunny weather). Such a $1.1-\mathrm{dB}$ power penalty chiefly results from the atmospheric attenuation because of $500 \mathrm{~m}$ free-space transmission and coupling loss for coupling laser light on the fibre ferrule ${ }^{27,28}$. Through $500-\mathrm{m}$ free-space transmission, laser light must be fitted into the fibre ferrule to avoid large coupling loss. Furthermore, it is to be observed that the BER performances of $x$-polarisation (with/without uplink PAM4 signals) and $y$-polarisation (with uplink PAM4 signals) are nearly the same. These show that there is no correlation between BER performance and polarisation state, and there is no correlation between downlink and uplink transmissions due to $4 \mathrm{~nm}$ coarse wavelength spacing between the downstream/upstream adjacent wavelengths. Additionally, in moderate rain, the BER increases to a $10^{-6}$ order of magnitude due to atmospheric attenuation from the moderate rain. In heavy rain, the BER performance severely degrades to a $10^{-3}$ order of magnitude due to high atmospheric attenuation from the heavy rain. The performance of FSO communication is highly dependent of the atmospheric channel condition. When the atmospheric channel condition is poor, then a transmitted laser light is significantly influenced by atmospheric turbulence. The inhomogeneity in the temperature and pressure over the atmospheric channel changes the refractive index of the atmosphere and brings on atmospheric attenuation ${ }^{29-31}$. Through $500 \mathrm{~m}$ free-space transmission, the atmospheric attenuation coefficient changes from $0.0042 \mathrm{~dB} / \mathrm{m}$ (sunny weather) to $0.025 \mathrm{~dB} / \mathrm{m}$ (heavy rain). The atmospheric attenuation coefficient $(\sigma)$ can be expressed $\mathrm{s}^{31}$ :

$$
\sigma=\frac{3.91}{V}\left(\frac{\lambda}{550 \mathrm{~nm}}\right)^{-q}
$$

where $V$ is the visibility, $\lambda$ is the wavelength, and $q$ is the size distribution of the scattering particles. In sunny weather, a $500-\mathrm{m}$ free-space transmission provides high link accessibility because of slight atmospheric attenuation. In heavy rain, a 500-m free-space transmission provides low link accessibility because of heavy atmospheric attenuation. In extremely heavy rain, however, a 500-m free-space transmission will be interrupted due to extremely high atmospheric attenuation coefficient of $0.061 \mathrm{~dB} / \mathrm{m}$.

Figure $2 \mathrm{~b}-\mathrm{e}$ exhibit the eye diagrams of $56 \mathrm{Gbit} / \mathrm{s}$ PAM4 signal in BTB state ( $x$-polarisation) and that over 25-km SMF transport with 500-m free-space transmission ( $x$-polarisation) under different weather conditions. In BTB sate, open eye diagrams are attained with $10^{-9}$ BER operation (Fig. 2b). In the state over 25-km SMF transport with 500 -m free-space transmission (sunny weather), a clear eye pattern is observed with $10^{-9}$ BER operation (Fig. 2c). Over 25-km SMF transport with 500-m free-space transmission (moderate rain), the eye diagrams are blurred with $10^{-6}$ BER operation (Fig. 2d). Over 25-km SMF transport with 500-m free-space transmission (heavy rain), however, the eye diagrams are closed with $10^{-3}$ BER operation (Fig. 2e).

BER performances of $224 \mathrm{Gbit} / \mathrm{s}$ PAM4-based fibre-FSO converged system (uplink transmission) in sunny weather under different scenarios and eye diagrams of $56 \mathrm{Gbit} / \mathrm{s}$ PAM4 signal in different conditions. Figure 3a exhibits the BER performances of $224 \mathrm{Gbit} / \mathrm{s}$ PAM4-based fibre-FSO 


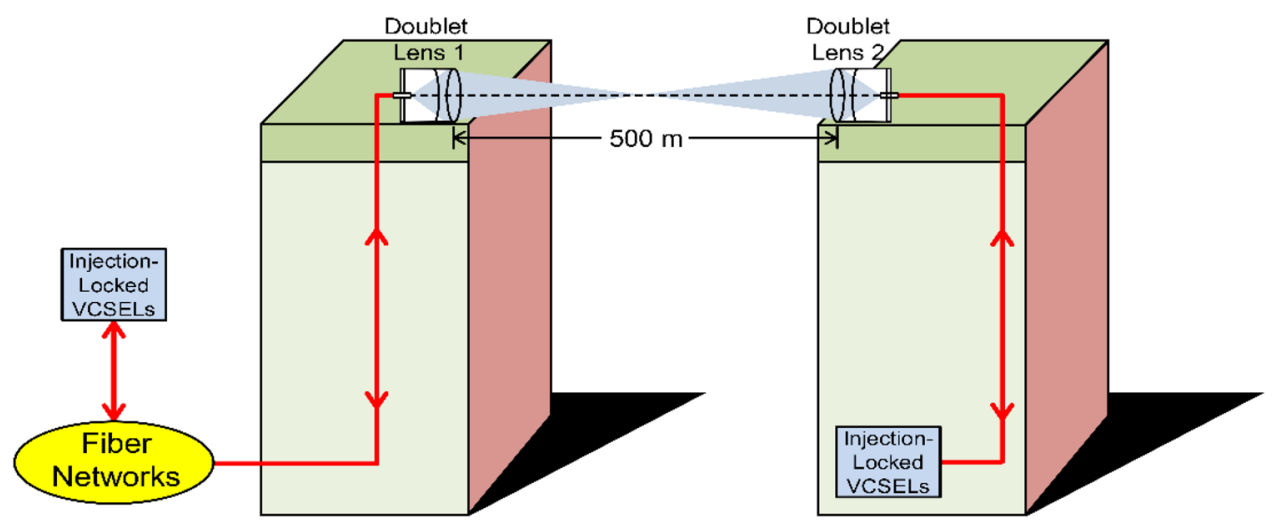

Figure 4. The layout of the doublet lenses-based two-way fibre-FSO converged system.

converged system (uplink transmission) in sunny weather under the scenarios of BTB ( $y$-polarisation), over 500-m free-space transmission ( $y$-polarisation), over 500-m free-space and $25-\mathrm{km}$ SMF transmissions $(x$ - and $y$-polarisations), over 600-m free-space and 25-km SMF transmissions ( $y$-polarisation), over 500-m free-space and $30-\mathrm{km}$ SMF transmissions ( $y$-polarisation), as well as over $500-\mathrm{m}$ free-space and $35-\mathrm{km}$ SMF transmissions ( $y$-polarisation). When the BER is $10^{-9}$, there exists $1.1 \mathrm{~dB}$ power penalty between the scenarios of BTB ( $y$-polarisation) and 500-m free-space transmission ( $y$-polarisation). This $1.1 \mathrm{~dB}$ power penalty is due to the atmospheric attenuation caused by $500-\mathrm{m}$ free-space transmission and coupling loss for doublet lens 1 coupling laser beam on the fibre ferrule. And further, as the BER is $10^{-9}$, there exists $5.7 \mathrm{~dB}$ power penalty between the scenarios of $500-\mathrm{m}$ free-space transmission ( $y$-polarisation) and $500-\mathrm{m}$ free-space with $25-\mathrm{km}$ SMF transmission ( $x$ - and $y$-polarisations). Such $5.7 \mathrm{~dB}$ power penalty is mainly due to the fibre dispersion because of $25-\mathrm{km}$ SMF transport. Additionally, it is to be found that the BER performances of $x$-polarisation (with downlink PAM4 signals) and $y$-polarisation (with/without downlink PAM4 signals) are almost the same. Furthermore, to have more associations with the free-space transmission distance, SMF length, and BER performance, we extend the free-space transmission distance and SMF length respectively to evaluate the BER performance. As the free-space transmission attains 600-m (600-m free-space $+25-\mathrm{km} \mathrm{SMF),} \mathrm{BER} \mathrm{increases} \mathrm{to} 10^{-7}$ due to more atmospheric attenuation and larger coupling loss. As the laser is delivered over $600-\mathrm{m}$ free-space transmission, it is difficult to completely concentrate the laser beam on the fibre ferrule due to laser's natural expansion, resulting in a large coupling loss. In addition, as the SMF length increases to $30 \mathrm{~km}(500-\mathrm{m}$ free-space $+30-\mathrm{km} \mathrm{SMF})$, BER performance degrades to a $10^{-7}$ order of magnitude due to more RB noise. As the SMF length further increases to $35 \mathrm{~km}$ (500-m free-space + 35-km SMF), BER performance severely degrades to a $10^{-5}$ order of magnitude due to higher RB noise. Because both downstream and upstream $256 \mathrm{Gbit} / \mathrm{s}$ PAM4 data signals are transmitted by the same SMF length, the RB noise thereby restricts the downstream and upstream transmission performances. When the SMF length is less than $25 \mathrm{~km}$, lower RB noise leads to better BER performance. Whereas as the SMF length exceeds $25 \mathrm{~km}$, higher RB noise results in poorer BER performance ${ }^{19-21}$. In long-haul lightwave transport systems $(>40 \mathrm{~km})$, the impairment is dominated by fiber dispersion-induced distortion. Given that the maximum SMF length in this demonstration is only $35 \mathrm{~km}(<40 \mathrm{~km})$, the impairment is thereby dominated by RB noise rather than fiber dispersion-induced distortion. Regarding eye diagrams, an open PAM4 eye diagram is attained (Fig. 3b) under the condition through 500-m free-space transmission ( $y$-polarisation). A clear PAM4 eye diagram is obtained (Fig. 3c) under the condition through 500-m free-space and 25-km SMF transmissions ( $y$-polarisation). Whereas a blurred PAM4 eye diagram is acquired (Fig. $3 \mathrm{~d}$ ) under the condition through $600-\mathrm{m}$ free-space and 25-km SMF transmissions ( $y$-polarisation).

\section{Discussion}

The layout of the doublet lenses-based two-way fibre-FSO converged system is presented in Fig. 4. Doublet lens emits and receives optical signals wirelessly to integrate fibre networks and FSO communications. Two doublet lenses are placed on the rooftops of two mansions to transmit/receive lasers through free-space in a two-way communication. Doublet lens is composed of one concave lens and one convex lens, with focal length and diameter of 150 and $50.8 \mathrm{~mm}$. Since the numerical aperture of the optical fiber is 0.14 , the laser light's diameter $(d)$ can be calculated as $42 \mathrm{~mm}[2 \times(150 \times 0.14)=42]$. The doublet lens 1's diameter $(50.8 \mathrm{~mm})$ is larger than laser light's diameter $(42 \mathrm{~mm})$ to make an FSO communication workable. The corresponding beam radius $(r)$ is given by

$$
r=\frac{2.3}{S F C \times 2 \pi}=3.6(\mu m)
$$

where $S F C$ is the spatial frequency cutoff. Thus, the laser light's divergent angle $(\theta)$ can be calculated as:

$$
\theta=\frac{3.6(\mu \mathrm{m})}{150(\mathrm{~mm})}=2.4 \times 10^{-5}
$$




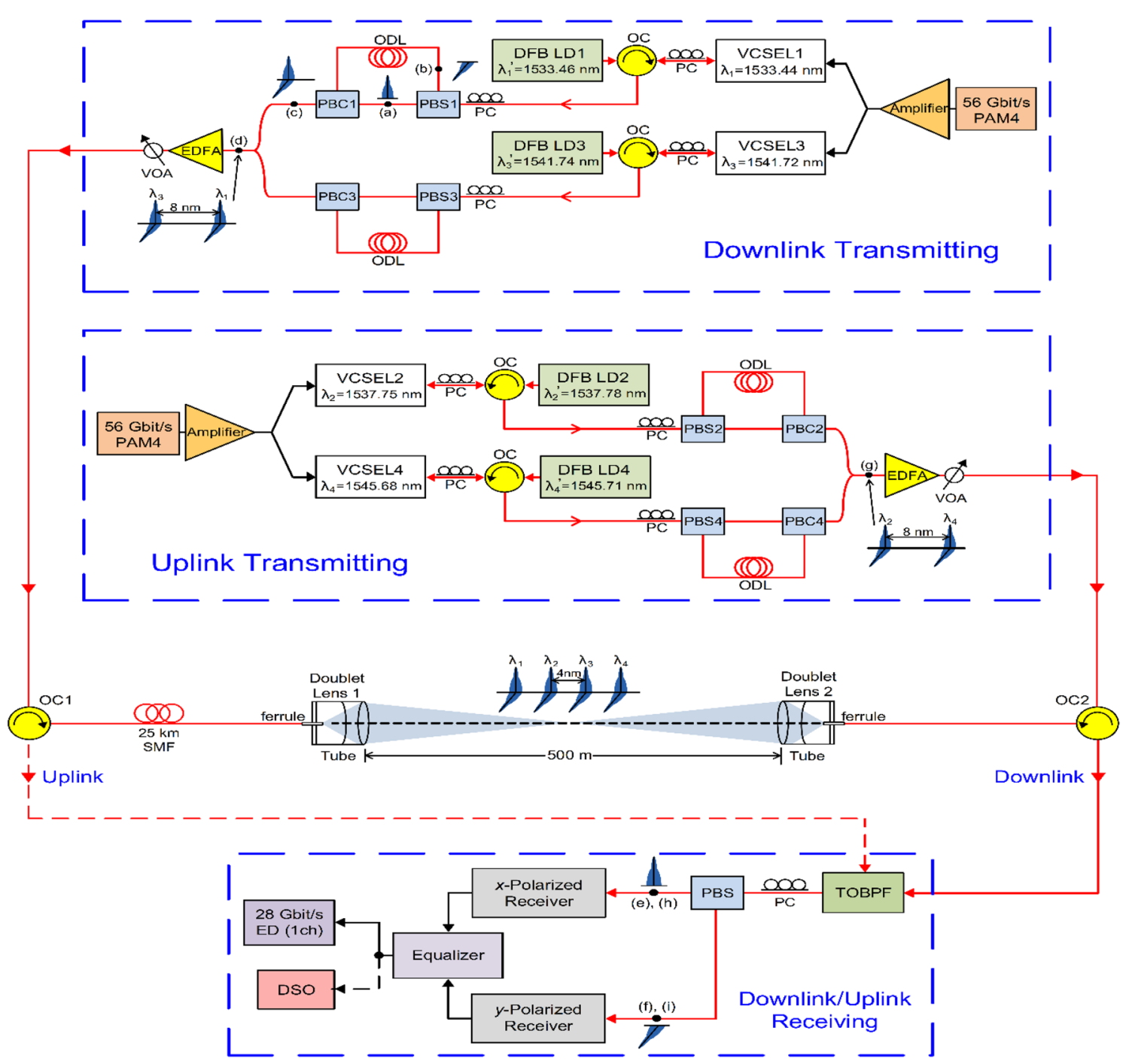

Figure 5. Framework of the demonstrated two-way 224-Gbit/s PAM4-based fibre-FSO converged system adopting injection-locked VCSELs with polarisation-multiplexing mechanism.

Over an $L$-m free-space link, the laser light's diameter $\left(d_{L}\right)$ must be smaller than doublet lens 2's diameter $\left(d_{L}<50.8 \mathrm{~mm}\right)$ to avoid large coupling loss and collect more laser light:

$$
d_{L}=\sqrt{d^{2}+(2 \theta L)^{2}}=\sqrt{42^{2}+(0.048 L)^{2}}<50.8
$$

$L$ is obtained as $595.4 \mathrm{~m}$, indicating the maximum free-space link is $595.4 \mathrm{~m}$. The free-space link in this demonstration is $500 \mathrm{~m}(<595.4 \mathrm{~m})$, which meets the free-space link demand.

\section{Methods}

Framework of the demonstrated two-way 224-Gbit/s PAM4-based fibre-FSO converged system adopting injection-locked VCSELs with polarisation-multiplexing mechanism over $25-\mathrm{km}$ SMF transport with 500-m free-space transmission. The framework of the demonstrated two-way 224-Gbit/s PAM4-based fibre-FSO converged system adopting injection-locked VCSELs with polarisation-multiplexing mechanism over 25-km SMF transport with 500-m free-space transmission is exhibited in Fig. 5. For downlink transmission, the 56 Gbit/s PAM4 signal created from the PAM4 signal generator is enhanced by a wideband amplifier, split by a $1 \times 2$ RF splitter, and inputted into VCSEL1 and VCSEL3. The light emitted from a DFB LD injects into a VCSEL (DFB LD1/1533.46 $\mathrm{nm} \rightarrow$ VCSEL1/1533.44 nm, DFB LD3/1541.74 $\mathrm{nm} \rightarrow$ VCS EL3/1541.72 nm) by utilizing an optical circulator (OC) with a polarisation controller (PC). After that, the 56 Gbit/s optical PAM4 signal is separated into $x$ - [inset (a)] and $y$-polarized [inset (b)] signals by a PC with a PBS. Then, these two polarized optical signals are combined by a polarisation beam combiner (PBC) [inset (c)]. If two polarisations are not separated by a PBS and then not combined by a PBC, an optical PAM4 signal with two orthogonal polarisations cannot be obtained. Given that the group velocities of $x$ - and $y$-polarized signals are 
different, one of the optical paths deploys an optical delay line to make up for the phase (optical path) difference between the two polarized signals. Two optical PAM4 signals with $x$ - and $y$-polarisations are coupled by a $2 \times 1$ optical coupler [inset (d)], promoted by an erbium-doped fibre amplifier, adjusted by a variable optical attenuator (VOA), and then delivered over 25-km SMF and 500-m free-space by two OCs (OC1 and OC2). Two doublet lenses at the transmitting/receiving site are utilized to implement a 500-m free-space transmission. Afterward, two integrated optical PAM4 signals with $x$ - and $y$-polarisations go through an OC2 and a TOBPF with $0.56 \mathrm{~nm}$ 3 - $\mathrm{dB}$ bandwidth, and deliver through a PC with a PBS to separate $x$ - and $y$-polarized optical PAM4 signals. Then, the $x$-polarized carrier with a 56-Gbit/s optical PAM4 signal [inset (e)] is sent to an $x$-polarized receiver. After electrical equalization, the PAM4 signal is calculated by BER parameter using a 28-Gbit/s error detector (ED). And further, the $56 \mathrm{Gbit} / \mathrm{s}$ PAM4 signal's eye diagrams are caught using a digital storage oscilloscope (DSO). Similarly, the $y$-polarized carrier with a 56-Gbit/s optical PAM4 signal [inset (f)] is supplied in a $y$-polarized receiver. After equalization, the PAM4 signal undergoes a real-time BER measurement using an ED. Moreover, a DSO is used to catch the eye diagrams.

For uplink transmission, the $56 \mathrm{Gbit} / \mathrm{s}$ PAM4 signal is driven via a broadband amplifier, divided by a $1 \times 2$ power divider, and supplied to VCSEL2 and VCSEL4. The light emitted from a DFB LD is injected into a VCSEL $(D F B ~ L D 2 / 1537.78 \mathrm{~nm} \rightarrow$ VCSEL2/1537.75 nm, DFB LD4/1545.71 nm $\rightarrow$ VCSEL4/1545.68 nm) through an OC integrated with a PC. Successively, the $56 \mathrm{Gbit} / \mathrm{s}$ optical PAM4 signal is divided into two parts with orthogonal states using a PC with a PBS. To offset the phase difference between the two optical paths, one of the optical paths deploys an optical delay line. Two optical PAM4 signals with $x$ - and $y$-polarisations are coupled by an optical coupler [inset (g)], magnified by an optical amplifier, controlled by a VOA, and communicated over $500-\mathrm{m}$ freespace and 25-km SMF by two OCs (OC2 and OC1). Next, two integrated optical PAM4 signals are circulated by an OC1, passed through a TOBPF, and delivered through a PC with a PBS to split $x$ - and $y$-polarized optical PAM4 signals. Next, the $x$-polarized/ $y$-polarized carrier with a 56 -Gbit/s optical PAM4 signal [inset (h)/inset (i)] is inputted into an $x$-polarized/ $y$-polarized receiver. After equalization, the performance of PAM4 signal is analyzed by BER parameter using a 28-Gbit/s ED. In addition, the 56 Gbit/s PAM4 signal's eye diagrams are taken by a DSO.

Received: 4 November 2021; Accepted: 21 December 2021

Published online: 10 January 2022

\section{References}

1. Corral, F. V., Cuenca, C. \& Soto, I. Design of an optical wireless network using free space optics technology (FSO), in 5G/6G networks environment. IEEE Intl Conf. on Autom./XXIV Congr. of the Chil. Assoc. of Automat. Contr. (ICA-ACCA), 1-5 (2021).

2. Li, C. Y. et al. A WDM PAM4 FSO-UWOC integrated system with a channel capacity of $100 \mathrm{Gbit} / \mathrm{s}$. IEEE/OSA J. Lightwave Technol. 38, 1766-1776 (2020).

3. Vallejo, L., Ortega, B., Bohata, J., Zvanovec S. \& Almenar, V. Experimental photonic 40-90 GHz millimetre-wave signal generation and $10 \mathrm{~Gb} / \mathrm{s} 32$-QAM signal transmission over hybrid fiber/FSO $5 \mathrm{G}$ networks. 21st Intl. Conf. on Transparent Opt. Netw. (ICTON), 1-4 (2019).

4. Bohata, J. et al. 24-26 GHz radio over fiber and free space optics for fifth-generation systems. Opt. Lett. 43, 1035-1038 (2018).

5. Quirce, A., de Dios, C., Valle, A., Prior, E., Pesquera, L., Panajotov, K., Palací, J., Meissner, P. \& Acedo, P. VCSEL-based optical frequency combs: Study of its polarisation dynamics under gain switching and polarisation selective optical injection locking. Intl. Conf. on Transparent Opt. Netw. (ICTON), 1-4 (2018).

6. Sayyah, K. et al. Two-dimensional pseudo-random optical phased array based on tandem optical injection locking of vertical cavity surface emitting lasers. Opt. Express 23, 19405-19416 (2015).

7. Wu, H. W. et al. A PDM-based 128-Gbit/s PAM4-based fibre-FSO converged system with OBPFs for polarisation demultiplexing. Sci. Rep. 10, $1872(2020)$.

8. Wei, L. Y. et al. Tricolor visible-light laser diodes based visible light communication operated at $40.665 \mathrm{Gbit} / \mathrm{s}$ and $2 \mathrm{~m}$ free-space propagation. Opt. Express 27, 25072-25077 (2019).

9. Yeh, C. H. et al. 1.7 to $2.3 \mathrm{Gbps}$ OOK LED VLC transmission based on $4 \times 4$ color-polarisation-multiplexing at extremely low illumination. IEEE Photonics J. 11, 7904206 (2019).

10. Wu, B. et al. Polarisation-insensitive remote access unit for radio-over-fibre mobile fronthaul system by reusing polarisation orthogonal light waves. IEEE Photonics J. 8, 7200108 (2016).

11. Shen, S. et al. Polarisation-tracking-free PDM supporting hybrid digital-analog transport for fixed-mobile systems. IEEE Photon. Technol. Lett. 31, 54-57 (2019).

12. Liu, S. J., Yan, J. H., Tseng, C. Y. \& Feng, K. M. Polarisation-tracking-free PDM IF-over-fibre mobile fronthaul employing multiband DDO-OFDM. Conference on Lasers and Electro-Optics (CLEO), SF2F.3 (2016).

13. Yao, S., Chen, Y. W., Su, C. \& Chang, G. K. Ubiquitous coverage next generation access networks based on fibre-FSO converged system with OBI-free heterodyne detection. IEEE Photonics Society Summer Topical Meeting Series (SUM), 1-2 (2019).

14. Wei, J. L., Ingham, J. D., Cunningham, D. G., Penty, R. V. \& White, I. H. Performance and power dissipation comparisons between $28 \mathrm{Gbit} / \mathrm{s}$ NRZ, PAM, CAP and optical OFDM systems for data communication applications. IEEE/OSA J. Lightwave Technol. 30, 3273-3280 (2012).

15. Lu, H. H. et al. $800 \mathrm{Gbit} / \mathrm{s} / 200 \mathrm{~m}$ FSO link with a WDM-PAM4 scheme and SLM-based beam tracking technology. Opt. Lett. 46, 1269-1272 (2021).

16. Singh, M., Atieh, A., Grover, A. \& Barukab, O. Performance analysis of $40 \mathrm{~Gb} / \mathrm{s}$ free space optics transmission based on orbital angular momentum multiplexed beams. Alexandria Eng. J. https://doi.org/10.1016/j.aej.2021.10.043 (2021).

17. Singh, M. et al. Performance investigation of a high data rate mode division multiplexed-free space optics link under harsh weather conditions. Front. Phys. 9, 743545 (2021).

18. Singh, M. et al. A high-speed radio over free space optics transmission link under dust environment conditions employing hybrid wavelength- and mode- division multiplexing. Wirel. Netw. 27, 4875-4888 (2021).

19. Yeh, C. H., Guo, B. S., Gu, C. S., Chow, C. W. \& Lin, W. P. Use of same WDM channels in fibre network for two-way free space optical communication with Rayleigh backscattering interference alleviation. IEEE Access 7, 169571-169576 (2019).

20. Wang, C. H. et al. Rayleigh noise mitigation using single sideband modulation generated by a dual-parallel MZM for carrier distributed PON. IEEE Photon. Technol. Lett. 22, 820-822 (2010). 
21. Chowdhury, A., Chien, H. C., Huang, M. F., Yu, J. \& Chang, G. K. Rayleigh backscattering noise-eliminated 115-km long-reach two-way centralized WDM-PON with 10-Gbit/s DPSK downstream and remodulated 2.5-Gbit/s OCS-SCM upstream signal. IEEE Photon. Technol. Lett. 20, 2081-2083 (2008).

22. Liu, H. et al. Turbulence-resistant FSO communication using a few-mode pre-amplified receiver. Sci. Rep. 9, 16247 (2019).

23. Khalighi, M. A. \& Uysal, M. Survey on free space optical communication: A communication theory perspective. IEEE Commun. Surv. Tuts. 16, 2231-2258 (2014)

24. Zhao, X. et al. Novel cascaded injection locking 1.55- $\mu \mathrm{m}$ VCSELs with $66 \mathrm{GHz}$ modulation bandwidth. Opt. Express 15, 1481014816 (2007).

25. Tsai, W. S., Lu, H. H., Su, C. W., Wang, Z. H. \& Li, C. Y. Centralized-light-source two-way PAM8/PAM4 FSO communications with parallel optical injection locking operation. IEEE Access 7, 36948-36957 (2019).

26. Lau, E. K., \& Wu, M. C. Amplitude and frequency modulation of the master laser in injection locking laser systems. IEEE International Topical Meeting on Microw. Photon. (MWP). MC-29, 142-145 (2004).

27. Moosavi, S. A. et al. Improvement of coupling efficiency in free-space optical communication with a multi-actuator adaptive lens. Opt. Lett. 44, 606-609 (2019).

28. Yeh, C. H. et al. Utilizing single lightwave for delivering baseband/FSO/MMW traffics simultaneously in PON architecture. IEEE Access 7, 138927-138931 (2019).

29. Nor, N. A. M. et al. Experimental investigation of all-optical relay-assisted $10 \mathrm{~Gb} / \mathrm{s}$ FSO link over the atmospheric turbulence channel. IEEE/OSA J. Lightwave Technol. 35, 45-53 (2017).

30. Kaushal, H. \& Kaddoum, G. Optical communication in space: Challenges and mitigation techniques. IEEE Commun. Surv. Tutor. 19, 57-96 (2017).

31. Kim, I. I., McArthur, B. \& Korevaar, E. Comparison of laser beam propagation at $785 \mathrm{~nm}$ and $1550 \mathrm{~nm}$ in fog and haze for optical wireless communications. Proc. SPIE 4214, 26-37 (2001).

\section{Acknowledgements}

This work was funded in part by the Ministry of Science and Technology of Taiwan (MOST 110-2221-E-027068-MY3) and in part by the Qualcomm Technologies, Inc. (NAT-457595).

\section{Author contributions}

H.-H.L., C.-Y.L., and W.-S.T. contributed to the experiment design. P.-S.C., Y.-Y.L., Y.-T.C., C.-X.L., and T.K. contributed to the experimental construction and measurement. H.-H.L., C.-Y.L., and W.-S.T. contributed to the data analysis. H.-H.L., C.-Y.L., and P.-S.C. contributed to the manuscript writing.

\section{Competing interests}

The authors declare no competing interests.

\section{Additional information}

Correspondence and requests for materials should be addressed to H.-H.L.

Reprints and permissions information is available at www.nature.com/reprints.

Publisher's note Springer Nature remains neutral with regard to jurisdictional claims in published maps and institutional affiliations.

(c) (i) Open Access This article is licensed under a Creative Commons Attribution 4.0 International License, which permits use, sharing, adaptation, distribution and reproduction in any medium or format, as long as you give appropriate credit to the original author(s) and the source, provide a link to the Creative Commons licence, and indicate if changes were made. The images or other third party material in this article are included in the article's Creative Commons licence, unless indicated otherwise in a credit line to the material. If material is not included in the article's Creative Commons licence and your intended use is not permitted by statutory regulation or exceeds the permitted use, you will need to obtain permission directly from the copyright holder. To view a copy of this licence, visit http://creativecommons.org/licenses/by/4.0/.

(C) The Author(s) 2022 